\title{
The Dietary Pattern of Pregnant Women Attending Ante Natal Clinic in a Tertiary Health Facility Centre in Nigeria
}

\author{
Ibiyemi Olasunbo Olayiwola ${ }^{1}$, Samson Ayo Deji ${ }^{2,}$, , Daniel O. Adesope ${ }^{3}$, Olawale O. Ajayi ${ }^{3}$, \\ Adebayo F. Adisa ${ }^{3}$, Adeola S. Akinola ${ }^{3}$, Folarin P. Akinlo ${ }^{3}$ \\ ${ }^{1}$ Department of Nutrition and Dietetics, Federal University of Agriculture, Abeokuta, Nigeria \\ ${ }^{2}$ Department of Epidemiology and Community Health, College of Medicine, Ekiti State University, Ado Ekiti, Nigeria \\ ${ }^{3}$ Department of Community Health, Obafemi Awolowo University, Ile Ife, Nigeria
}

Email address:

samdeji3@hotmail.com (S. A. Deji)

\section{To cite this article:}

Ibiyemi Olasunbo Olayiwola, Samson Ayo Deji, Daniel O. Adesope, Olawale O. Ajayi, Adebayo F. Adisa, Adeola S. Akinola, Folarin P. Akinlo. The Dietary Pattern of Pregnant Women Attending Ante Natal Clinic in a Tertiary Health Facility Centre in Nigeria. European Journal of Preventive Medicine. Vol. 3, No. 3, 2015, pp. 75-79. doi: 10.11648/j.ejpm.20150303.17

\begin{abstract}
Nutritional status of pregnant women is very crucial to the wellbeing of the unborn child. Good dietary pattern among pregnant women reduces the rate of maternal and infant mortality which is still a big problem in developing countries. The study assessed food and dietary pattern, energy intake and awareness of the importance of taking adequate micronutrients during pregnancy. A total of 400 pregnant women attending a routine antenatal clinic of the Obafemi Awolowo University Teaching Hospital Ile Ife, Nigeria were recruited into the study by simple random sampling method on different clinic days. Structured interviewer administered questionnaires were used to elicit data on socio-demographic characteristics, anthropometric indices and 24 hour dietary recall which were used to assess the dietary pattern and nutritional status of the respondents. Seventy two per cent of the respondents were between the ages $25-34$ years, $91 \%$ were from monogamy family structure, $72 \%$ had tertiary education and $83 \%$ were aware of micronutrients intake. About $98 \%$ of respondents consumed on a daily basis, food rich in carbohydrate, vegetable and fruits but 39\% reported consuming protein rich diet such as fish and meat . Conclusively, the research confirmed that the dietary pattern of pregnant women showed they consumed more of carbohydrate diet compared to protein diet which is most important especially during pregnancy to improve their nutritional status and that of the unborn baby. More attention should be paid to nutritional education on the choice of food pregnant women eat, especially the less educated women and those with low income status.
\end{abstract}

Keywords: Diet, Pregnancy, Nutrition, Micronutrient

\section{Introduction}

Good nutrition with additional nutrients are required during pregnancy for the development of the foetus, good maternal health and also enhance effective breastfeeding after delivery. Adequate maternal weight status arising from good dietary pattern influences the health of both the mother and her child throughout the entire reproductive cycle, spanning from the time of conception and post delivery period of lactation. There is a relative paucity of data on maternal dietary pattern and its association with weight gain in most parts of developing countries. In addition, little is known about the association between obesity and excessive weight gain in pregnancy (Erkkola and Virtanen, 1998), (Lyytikäinen, 2005), (Paturi and Pietinen, 2008). Studies have shown that among Finnish girls and women, intakes of vitamin D, folic acid and iron were relatively below the expected minimal level to achieve good nutritional status. (Barker, 1992).

The National Demographic Health Survey in Nigeria estimated that about $26 \%$ of women of childbearing age were malnourished largely due to poor dietary habits and more than $50 \%$ of child bearing age women in their pre pregnancy state have less than optimal nutritional status. (NDHS 2008) In view of these facts, the concept of poor nutrition during pregnancy resulting in childhood and adult disease has been generated by the foetal origin theory of adult diseases. (Godfrey, 2002). Factors such as poor quality of food, food restrictions due to superstitious beliefs, unhealthy feeding habits and inadequate knowledge of good dietary pattern have been well documented as variables associated with 
pregnancy outcome and weight gain during pregnancy (Campbell and Campbell 2008) This has resulted in morbidity and mortality among pregnant women with children born either weighing below or above the expected normal weight for a healthy new-born. Most congenital cardiovascular diseases among children and adulthood originate at the embryonic developmental stages where essential nutrient required for healthy foetus development are lacking because they are not transferred through the placental of the woman to the unborn baby due to consumption of poor quality diet deficient of such basic nutrients.(Gluckman, Hanson and Pinal 2005). On the other hand excessive consumption of food items such as carbohydrates, fatty and protein diet has resulted in overweight and obesity among some pregnant women. Excessive weight in pregnancy is associated with many disease conditions such as hypertension in pregnancy, diabetes mellitus, giving birth to big babies who may later in life have health challenges and cardiovascular diseases. These medical conditions may continue to the post-partum period. (Hasunen, Kalavainen and Talvia, 2004). This article focused on the dietary habits, awareness of the importance in using micronutrient by pregnant with a view of establishing the role of nutrition in pregnancy The specific objective of this study was to assess the dietary pattern of pregnant women attending an ante natal clinic in a tertiary health facility centre in Nigeria.

\section{Materials and Methods}

The study design was a descriptive cross sectional study with a target population of pregnant women in Nigeria. The study population were pregnant women attending their normal ante natal clinic during the period of the research work. The study was conducted at the ante natal clinic of Obafemi Awolowo University Teaching hospitals Complex Ile-ife, Osun state in the south western zone of Nigeria. The teaching hospital is affiliated with Obafemi Awolowo University Ile Ife. It is a tertiary health facility centre where complex medical cases are treated by well-trained specialist doctors in various fields of medicine including obstetrics and gynaecology a field of medicine that care for all women related health problems. Basically, tertiary level of health care delivery is offered at the centre. The Health facility receives referral of medical cases from neighbouring towns and villages for expert medical treatment.

\subsection{Sample Size}

Sample size was determined using the formula

$$
N=\frac{Z^{2}(p q)}{d^{2}}
$$

where

$\mathrm{n}$ is the sample size required

$\mathrm{z}$ is the standard normal variate, $95 \%$ is approximately 1.96

$\mathrm{p}$ is the prevalence of the attribute $\mathrm{q}$ is $(1-\mathrm{p})$

$\mathrm{d}$ is the precision $(0.05)$

In other to estimate this prevalence with $95 \%$ confidence and a precision of $10 \%$, a total of 384 pregnant women were needed. The sample size was rounded up to 400 of all pregnant women. (NDHS 2008)

Sampling Technique: Respondents who were pregnant women attending the ante natal clinic of the hospital were randomly recruited for the study at different clinic days. Four hundred women were randomly selected for the study

\subsection{Data Collection and Instrument}

Structured questionnaires were used to collect information on the Socio demographic characteristics, dietary pattern and 24 hour dietary recall.

\subsection{Data Analysis}

The data collected were coded and analysed with the aid of SPSS (Statistical Package for Social Sciences) software version 17.0 and relationships between variables were determined using Chi-square analysis. P-value of $<0.05$ was taken as significant.

\subsection{Ethical Considerations}

Ethical clearance was obtained from Research and Ethics Obafemi Awolowo University, Ile Ife prior to commencement of the study. Consent was obtained from the respondents. Participation in the study was completely voluntary and the respondents were assured of confidentiality.

\section{Results}

Table 1. Socio-demographic characteristics of respondents.

\begin{tabular}{lll}
\hline Variables & $\mathbf{n}=\mathbf{4 0 0} \mathbf{( 1 0 0 \% )}$ & $\mathbf{N}(\mathbf{1 0 0} \mathbf{)})$ \\
\hline Age (years) & & \\
$15-24$ & 56 & 14.1 \\
$25-34$ & 288 & 72.7 \\
$35-45$ & 52 & 13.1 \\
Religion & & \\
Christianity & 320 & 80.0 \\
Islam & 80 & 20.0 \\
Employment Type & & \\
Self-employed & 124 & 33.3 \\
Civil servant & 152 & 40.9 \\
Unemployed & 96 & 25.9 \\
Income per month in Nigeria Currency. & & \\
$<21000$ & 276 & 70.4 \\
21000-40000 & 48 & 12.2 \\
$41000-60000$ & 24 & 6.1 \\
$>60000$ & 44 & 11.2 \\
Educational level & & \\
No formal education & 12 & 3 \\
Primary & 4 & 1 \\
Secondary & 104 & 26 \\
Tertiary & 284 & 71 \\
Family Type & & 91.0 \\
Monogamy & 360 & 9.0 \\
Polygamy & 36 & \\
\hline
\end{tabular}

Key: * 
As revealed in table 1; majority of the respondents, $72.7 \%$ were between the age range of $15-45$ years. About $70.4 \%$ of respondents lived on a monthly income less than 20,000 naira equivalent of 100 dollars. Most of the respondents were civil servants, $32 \%$ and majority, $71 \%$, had tertiary education.

Table 2 , showed that $83.3 \%$ of the pregnant women were aware of the need to take micronutrient druing pregnancy. The highest source of information about the importance of micronutrient intake during pregnancy as reported by respondents in table 2 was from the hospitals (78\%).

Table 3 showed the dietary frequency pattern among respondents with food rich in micronutrients such as vegetables, fruits and fortified foods, being the major item consumed by respondents on daily basis, $100 \%$.Only $39 \%$ of the respondents reported to have consumed protein rich diet on daily basis while $11 \%$ reported consuming protein occasionally which is very significant.

Table 2. Awareness and sources of Awareness of micronutrient intake among pregnant women.

\begin{tabular}{lll}
\hline Variables & Frequency & Percentage (\%) \\
\hline $\begin{array}{l}\text { Aware of the necessity of micronutrient } \\
\text { intake during pregnancy }\end{array}$ & \\
Yes & 320 & 83.3 \\
No & 64 & 16.7 \\
Total & 384 & 100.0 \\
Sources of Information & & \\
Mass media & 16 & 4.9 \\
Hospital & 256 & 78 \\
Family/ Friends & 40 & 12.2 \\
Internet & 16 & 4.9 \\
Total & 318 & 100 \\
\hline
\end{tabular}

Table 3. Food consumption pattern of the respondents.

\begin{tabular}{lllll}
\hline Food list & Daily N (\%) & Once a Week N (\%) & Occasionally N (\%) & Remarks \\
\hline Carbohydrate (garri, bread, fufu, spaghetti, yam) & $392(98)$ & 4 & 0 & High \\
Protein (beans, egg, meat, fish) & $156(39.0)$ & $192(48)$ & $44(11.0)$ & Moderate \\
Fats \& Oil (groundnut oil, soyb8ean oil, palm oil) & $380(95.0)$ & $12(3.0)$ & $0(0.0)$ & High \\
Micronutrients (vegetables, water leaf, orange, banana) & $400(100)$ & $0(0.0)$ & $0(0.0)$ & High \\
\hline
\end{tabular}

The Energy intake ranged from 1111-11638 kcal/day.

Table 4. The Relationship Between Energy \& Dietary Intake.

\begin{tabular}{llll}
\hline \multirow{2}{*}{ Dietary Intake } & Energy & & High(>2700) \\
\cline { 2 - 4 } & Low(<2500) & Normal(2500-2700) & 228 \\
\hline Daily & 52 & 12 & 4 \\
Weekly & 4 & 0 & 232 \\
Total & 56 & 12 & 23 \\
\hline
\end{tabular}

Chi-Square (5.315), P-Value (0.021)

Table 5. The effect of socio demographic status on the energy intake.

\begin{tabular}{|c|c|c|c|c|c|}
\hline \multicolumn{4}{|l|}{ Energy } & \multirow{2}{*}{ Chi-Square Value } & \multirow{2}{*}{ Chi-Square P-Value } \\
\hline & Low $(<2500)$ & Normal (2500-2700) & High $(>2700)$ & & \\
\hline \multicolumn{6}{|l|}{ Education } \\
\hline Primary & 4 & 0 & 0 & \multirow{4}{*}{20.349} & \multirow{4}{*}{0.000} \\
\hline Secondary & 20 & 4 & 80 & & \\
\hline Tertiary & 44 & 8 & 232 & & \\
\hline Total & 68 & 12 & 312 & & \\
\hline \multicolumn{6}{|l|}{ Income } \\
\hline$<21000$ & 60 & 8 & 240 & \multirow{5}{*}{22.504} & \multirow{5}{*}{0.001} \\
\hline $21000-40000$ & 0 & 0 & 44 & & \\
\hline $41000-60000$ & 0 & 0 & 24 & & \\
\hline$>80000$ & 8 & 0 & 36 & & \\
\hline Total & 68 & 8 & 308 & & \\
\hline \multicolumn{6}{|l|}{ Age group } \\
\hline $15-19$ & 0 & 0 & 8 & \multirow{7}{*}{30.206} & \multirow{7}{*}{0.001} \\
\hline $20-24$ & 8 & 0 & 40 & & \\
\hline $25-29$ & 24 & 4 & 124 & & \\
\hline $30-34$ & 36 & 8 & 88 & & \\
\hline $35-39$ & 0 & 0 & 44 & & \\
\hline $40-45$ & 0 & 0 & 4 & & \\
\hline Total & 68 & 12 & 308 & & \\
\hline
\end{tabular}


Table 4 showed that the dietary intake had a significant effect on energy of pregnant women in 24 hours, with Pvalue of 0.021 which is less than 0.05 significant levels.

From table 5, on Education, the P-value obtained was 0.000 which was significant in the effect on Energy intake. On Income, the P-value obtained was 0.001 which is also significant on the effect on Energy intake. On Age, there was also a significant relationship to the intake of Energy.

Table 6. Energy intake pattern during three trimesters of respondents.

\begin{tabular}{llll}
\hline \multirow{2}{*}{ Trimester } & Energy & & \\
\cline { 2 - 4 } & Low $(<\mathbf{2 5 0 0})$ & Normal(2500-2700) & High $(>\mathbf{2 7 0 0})$ \\
\hline 1 & 0 & 0 & 4 \\
2 & 40 & 4 & 76 \\
3 & 28 & 8 & 228 \\
Total & 68 & 12 & 388 \\
\hline
\end{tabular}

Chi- square (30.901), P-value (0.000)

Table 6 showed that in the first trimester only 4 respondents had energy intake above $2700 \mathrm{Kcal}$, in the second trimester; 40 respondents had energy level below $2500 \mathrm{Kcal}$ and in the third trimester, only 8 of them had a normal energy intake. The P-value obtained from the effect of Trimesters on Energy was significant. $(\mathrm{P}<0.05)$.

\section{Discussion}

This study investigated dietary pattern during pregnancy as well as their energy intake at each trimester. Awareness of necessity to consume diet rich in micronutrients was also assessed. Quality and balanced diet becomes very crucial to the health of the woman during and after pregnancy. Previous documented studies have affirmed the fact that diet during and after pregnancy has significant impact on the mother and the baby several years after delivery. (Becker et al, 2006).

This study showed all respondents to have consumed food rich in micronutrients, carbohydrates, fats and oil adequate enough for their status as pregnant women. But food rich in proteins was poorly consumed and this is most needed during pregnancy for healthy development of the unborn baby and adequate maintenance of body tissue repairs. This is common in most African countries where majority of pregnant women cannot afford to buy high expensive protein rich diet such meat, fish etc due to poverty.

Age, education and income had positive relationships with the consumption of energy rich food. The same association was found earlier in pregnant women in the US. Nevertheless, the consumption of these foods items was also reported to be low in another study among pregnant women in the US, (Williams, Prevost and Wareham 2000).

The statistic from National Demographic and Health Survey of Nigeria (NDHS) 2008 reported that consumption of meat, fish, poultry, and egg increases with level of education and income status among women. The healthier food choices made as reported in the study was higher among the well educated pregnant women. Income status on one hand contribute to poor dietary habits of pregnant women while poor level of education contributes more. Most illiterate will rather spend their money purchasing large quantity rather than quality foodstuff.

Food rich in micronutrient intake among women have been characterized by regular use of vegetables and fruit, (Lyytikäinen, 2005), (Helakorpi , Patja and Prättälä, 2007), ( Nilsen and Vollset, 2006) as also seen in this study where all the respondents reported to have consumed micronutrient rich diet on daily basis.

Food rich in micronutrient intake are vital supplements supposed to complement the dietary intake of pregnant women because of the role micronutrient play in the developmental milestones of the baby even after delivery. When this vital micronutrients are not consumed in their right proportion they can result in severe birth defects such as congenital heart diseases, spinal bifida, slow mental development among others. It is therefore most advocated that pregnant women should take these food items throughout the period of their pregnancy. Our study revealed that $83.3 \%$ of the respondents demonstrated awareness of the importance of micronutrient intake during pregnancy as well as $100 \%$ reported to have consumed food rich in micronutrients daily. This proportion is parallel to the results in an earlier study among Finnish pregnant women where $70 \%$ of them reported use of at least one dietary supplement. The prevalence of women who do not consume diet rich in micronutrient is even higher among non-pregnant Finnish women (Helakorpi, Patja and Prättälä, 2007).

In our study it showed that maternal age, education and income are associated with energy intake during pregnancy which shows a significant positive relationship between these variables. The higher the maternal age the more energy they consume, the higher the educational level; the more the energy intake also the more money they earn the higher the energy intake among the pregnant women. It was noticed that the women with low educational status were those that earn less and was found to have low energy intake.

\section{Conclusions and Recommendation}

Findings of the present study suggest that healthy food choices are relatively common among the pregnant women with high energy intake and increase micronutrient intakes during pregnancy. However, a significant percentage of them consumed less of protein rich diet during pregnancy. The awareness of the respondents about the need to consume micronutrient rich diet was high and the source of their information was from the hospital.

Eight dietary patterns were identified among these pregnant women providing a meaningful interpretation of their dietary habits. The present results strengthen the assumption that young and less educated women with those of low income are at risk of having an inadequate dietary 
pattern. A strong association exist between the maternal age, education, level of income and nutritional status. Thus, young and less well educated women and those with low income in particular should be a special target group as regards nutrition education, with emphasis on well-planned adequate meals and more precise advice on adequate intake of micronutrient rich diet during pregnancy. This is important for optimal pregnancy outcome, successful breastfeeding, and for the prevention of obesity or other nutrition related disease in later life, among both mothers and children. Dietary patterns may be a useful tool for risk group identification and offer a framework for further research concerning diet and health outcomes among mothers and their children.

\section{References}

[1] Barker D (1992) Fetal and Infant Origins of Adult Disease. London, British Medical Journal Books.

[2] Becker W, Alexander J, Andersen S, Aro A, Thorsdottir I (2006) Nordic nutrition recommendations. UgeskrLaeger 168: $76-77$.

[3] Erkkola M, Virtanen SM (1998) Folate, Vitamin D and iron: Low among pregnant Finnish women. Eur J ClinNutr 52: 742-748.

[4] Gluckman P D, Hanson M A \& Pinal C (2005) The developmental origins of adult disease. Maternal Child Nutr 1: $130-141$
[5] Godfrey K M (2002) The role of the placenta in fetal programming-a review. Placenta 23: S20-S27.

[6] Hasunen K, Kalavainen M, Talvia S (2004) The Child, Family and Food - Nutrition recommendations for infants and young children as well as pregnant and breastfeeding mothers (in Finnish).

[7] Helakorpi S, Patja K, Prättälä R (2007) Health behaviour and health among the Finnish adult population. Helsinki, National Public Health Institute.

[8] Lyytikäinen A (2005) Food consumption and nutrient intakes with a special focus on milk product consumption in early pubertal girls in Central Finland. Public Health Nutr 8: 284 289.

[9] Nilsen R M, Vollset SE (2006) Patterns and predictors of folic acid supplement use among pregnant women: the Norwegian Mother and Child Cohort Study. Am J ClinNutr 84: 1134-114.

[10] Paturi M, Pietinen P (2008) The national FINDIET 2007 survey. Publications of the National Public Health Institute, B23/2008. Helsinki, Helsinki University Press.

[11] Slattery M L, Boucher KM \& Ma KN (1998) Eating patterns and risk of colon cancer. Am J Epidemiol 148: 4-16.

[12] Voyles L M, Turner RE (2000) High levels of retinol intake during the first trimester of pregnancy result from use of overthe counter vitamin/mineral supplements. J Am Diet Assoc 100: $1068-1070$.

[13] Williams D E, Prevost AT \& Wareham NJ (2000) A crosssectional study of dietary patterns with glucose intolerance and other features of the metabolic syndrome. Br J Nutr 83: 257-266. 